\title{
A Simpler Minimal
}

\author{
Mauro Del Giglio ${ }^{1}$, Gabriele Tamagnini ${ }^{1}$, Raoul Biondi ${ }^{1}$, and Michele Di Mauro ${ }^{2}$ \\ ${ }^{1}$ Villa Torri Hospital \\ ${ }^{2}$ Maastricht UMC+
}

July 27, 2020

\begin{abstract}
Minimally Invasive Aortic Valve Replacement is not just a metric of the incision, but rather a holistic approach to minimize the surgical trauma: the technique should reproduce the gold-standard conventional procedure in terms of safety, effectiveness and operative times through a small and different incision. Moreover, the procedure should be simple and reproducible in every Center all over the world. In our experience, we rely more on surgical skills and technique optimization, rather than CT-scan planning: definitely, the pre-operative imaging is helpful in the beginning of the experience to rule out difficult cases.
\end{abstract}

\section{Letter to the Editor}

Dear Editor,

Karel Van Praet et al. recently published the interesting "RALT approach" (1) for minimally invasive aortic valve replacement.

As stated by the authors in the abstract, the CT scan ismandatory for planning a MiAVR. We have treated more than 1200 patients with a total central cannulation MiAVR approach $(2,3)$ and in our opinion this message is misleading: the non-invasive anatomical assessment might be useful in the beginning of the experience to rule out difficult cases, especially the ones who have got a longmini-thoracotomy to aortic annulus distance; moreover, the surgical access site selection does not require a CT scan, the third being the right intercostal space (rarely if not, the surgeon can easily change it to the second from the same skin incision). In the real world, a dedicated CT scan means technological skills, time and financial resources and they might not be found at once in every Center who wants to start a MiAVR program; on the contrary this surgical approach has to be direct and simplified, made suitable for everyone (4). The video assistance, the peripheral cannulation and the transcutaneous clamping allow to avoid any need of costal spreading and occasional rib injuries: the camera should be the surgeon's eyes and the mini-thoracotomy only the access to bring the valve in. Minimally invasive surgery is a skill to reduce the surgical burden: the use of central cannulation reduces surgical trauma and avoids potential complications due to femoral cannulation such as dissection of the femoral vessel and the aorta, ischemia of the ipsilateral limb, cerebrovascular and renal events (5), allowing a more physiological perfusion flow. Furthermore, in our experience, the venous central cannulation optimizes the exposure (simply with a wire which gently pull away to right auricula from the aortic root) and also it reduces the shear stress, since that does not require a vacuum assistance in most cases; we came up with a slightly modified cannula, which can be shaped to engage easily the inferior cava vein. The Authors refer to a SLL-PEEP technique to inflate the left lung and push the aorta towards the surgical access, in case of suboptimal exposition: we rely on pericardial stitches rather than inflating the left lung, in order to avoid any risk of barotrauma.

In our opinion, MiAVR has to reproduce the gold-standard conventional procedure in terms of safety, effectiveness and especially operative times through a more respectful approach; the non-invasive CT-scan assessment might be helpful, especially in the beginning of the experience. 


\section{References}

1. Van Praet KM, van Kampen A, Kofler M, Richter G, Sündermann SH, Meyer A, et al. Minimally invasive surgical aortic valve replacement: The RALT approach. Journal of Cardiac Surgery. 2020 Jul 9.

2. Mikus E, Calvi S, Campo G, Pavasini R, Paris M, Raviola E, et al. Full Sternotomy, Hemisternotomy, and Minithoracotomy for Aortic Valve Surgery: Is There a Difference? The Annals of Thoracic Surgery. 2018 Dec;106(6):1782-8.

3. Mikus E, Turci S, Calvi S, Ricci M, Dozza L, Del Giglio M. Aortic valve replacement through right minithoracotomy: is it really biologically minimally invasive? The Annals of Thoracic Surgery. 2015 Mar;99(3):82630 .

4. Sanad M, Beshir H. Minimally invasive aortic valve replacement with central cannulation: A cost-benefit analysis in a developing country. Cardiothorac Surg. The Cardiothoracic Surgeon; 2020 Mar 2;28(1):1-11.

5. Bedeir K, Reardon M, Ramchandani M, Singh K, Ramlawi B. Elevated Stroke Risk Associated With Femoral Artery Cannulation During Mitral Valve Surgery. Semin Thorac Cardiovasc Surg. 2015;27(2):97103. 